Revue des sciences de l'éducation

Compétences à mobiliser pour la compréhension et l'interprétation de manuels d'histoire du secondaire au Québec Competencies needed to understand and interpret Quebec secondary level history textbooks

Competencias a movilizar para la comprensión y la interpretación de los libros de historia en la secundaria en Quebec

\author{
Suzanne-G. Chartrand
}

Volume 35, numéro 2, 2009

Les manuels scolaires : réformes curriculaires, développement professionnel et apprentissages des élèves

URI : https://id.erudit.org/iderudit/038728ar

DOI : https://doi.org/10.7202/038728ar

Aller au sommaire du numéro

Éditeur(s)

Revue des sciences de l'éducation

ISSN

0318-479X (imprimé)

1705-0065 (numérique)

Découvrir la revue

Citer cet article

Chartrand, S.-G. (2009). Compétences à mobiliser pour la compréhension et l'interprétation de manuels d'histoire du secondaire au Québec. Revue des sciences de l'éducation, 35(2), 37-58. https://doi.org/10.7202/038728ar

\section{Résumé de l'article}

Une analyse de la mise en discours de deux chapitres de deux manuels d'histoire générale conçus pour des élèves québécois de 13-14 ans a été menée afin de connaître quelles compétences ces élèves doivent mobiliser en lecture pour que ces manuels constituent un réel outil d'apprentissage, a fortiori lorsqu'ils se présentent comme des substituts de l'enseignant. Il en ressort que, sans un étayage systématique de la part des enseignants pour assurer la compréhension et l'interprétation des élèves, ces manuels ne peuvent jouer leur rôle, car ils présentent de trop nombreux obstacles pour de jeunes lecteurs. 


\title{
Compétences à mobiliser pour la compréhension et l'interprétation de manuels d'histoire du secondaire au Québec*
}

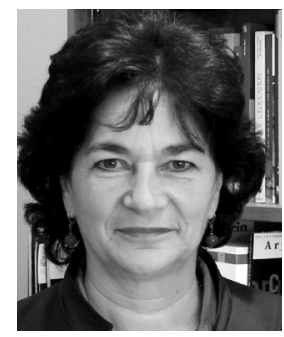

Suzanne-G. Chartrand, didacticienne du français Université Laval

\begin{abstract}
RÉSUMÉ - Une analyse de la mise en discours de deux chapitres de deux manuels d'histoire générale conçus pour des élèves québécois de 13-14 ans a été menée afin de connaître quelles compétences ces élèves doivent mobiliser en lecture pour que ces manuels constituent un réel outil d'apprentissage, a fortiori lorsqu'ils se présentent comme des substituts de l'enseignant. Il en ressort que, sans un étayage systématique de la part des enseignants pour assurer la compréhension et l'interprétation des élèves, ces manuels ne peuvent jouer leur rôle, car ils présentent de trop nombreux obstacles pour de jeunes lecteurs.
\end{abstract}

MOTS CLÉS · manuel, enseignement de l'histoire, compréhension, lecture, compétence en lecture.

\section{Introduction et problématique}

D'une discipline à l'autre, la mise en discours des objets de savoir prend des formes différentes, puisque chacune possède son langage et ses codes (Ginsburger-Vogel, 1988). Les textes étudiés dans les cours de sciences, d'histoire, de mathématiques et de français présentent une grande variété de statuts, de structures, de styles et d'enjeux pour la compréhension des élèves.

Didacticienne du français, nous avons mené une recherche sur les activités de lecture et d'écriture dans les classes d'histoire et de sciences du secondaire québé-

\footnotetext{
* Subventionnée par le Fonds québécois de recherche sur la société et la culture (FQRSC) et le ministère de l'Éducation du Québec (MÉQ) et le Conseil de recherches en sciences humaines du Canada (CRSH), de 2004 à 2007, la recherche Scriptura porte sur les activités de lecture et d'écriture, comme médiations de l'apprentissage, en classes de sciences et d'histoire. En outre, une partie de cette étude a bénéficié du soutien financier de la Faculté des sciences de l'éducation de l'Université Laval (subvention BDR-FSÉ).
} 
cois. Cette recherche comprenait une enquête dans 50 écoles secondaires de la grande région de Québec, dont la population est représentative de celle de l'ensemble du Québec. Des quelque 360 enseignants d'histoire et de sciences sollicités, 100 ont répondu à un questionnaire portant, entre autres, sur les activités de lecture et d'écriture qu'ils font en classe. Un autre questionnaire a été administré à 1150 élèves de classes mixtes de $2^{e}$ et de $4^{e}$ secondaire (617 filles et 533 garçons) d'écoles de différents milieux et offrant des programmes d'études variés (Blaser, 2007; Chartrand et Blaser, 2006; Chartrand, Blaser et Gagnon, 2006).

Nous présenterons ici les résultats qui concernent les cours d'histoire. À ce propos, nous voulions savoir si le manuel d'histoire, principal support utilisé dans les classes, est conçu et rédigé de manière à ce que les élèves puissent le comprendre avec une relative autonomie. Autrement dit, les modes de mise en discours dans les manuels sont-ils adaptés à de jeunes lecteurs, dont plus du quart ont des retards en lecture à la fin du primaire (Baudelot, Cartier et Detrez, 1999; Garon, 2004; Pierre, 2003a, 2003b)?

D'après l'enquête Scriptura, selon les élèves de $2^{\mathrm{e}}$ et de $4^{\mathrm{e}}$ secondaire, en 20042005, dans le cours d'histoire, les textes souvent / très souvent lus sont, dans l'ordre décroissant: 1$)$ des notes de cours $(78 \%) ; 2$ ) des textes du manuel $(66 \%) ; 3)$ des textes trouvés sur Internet $(24 \%) ; 4)$ des articles de revues ou de journaux $(22 \%)$. Selon $73 \%$ des élèves, leur enseignant trouve important ou plutôt important de les faire lire en classe. Quant à $47 \%$ d'entre eux, ils disent ne jamais ou parfois trouver intéressant ce qu'ils lisent en histoire. Notons que, de tous les supports de textes utilisés en classe d'histoire, le manuel est celui qui est le moins apprécié des élèves participant à l'enquête, bien qu'il soit le plus utilisé selon eux et leurs enseignants. La recherche Scriptura a également permis d'observer quatre séquences d'enseignement de l'histoire, de 5 à 12 périodes, dans deux classes de $2^{\mathrm{e}}$ secondaire et dans deux de $4^{e}$ (Blaser, 2007). Il en est ressorti que les enseignants ont peu de pratiques d'étayage pour permettre la compréhension des manuels par leurs élèves et sousestiment les difficultés de ces outils.

Pour la partie de la recherche étudiée dans le présent article, en nous appuyant sur les recherches dans le domaine de la psycholinguistique (Morais, 1994), de la psychologie cognitive (Escalle et Magnan, 2003; Golder et Goanac'h, 1998; Pierre, 2003b) et de la didactique de la lecture (Giasson, 1995a, 2000; Grossmann, 1999; Préfontaine, Godard et Fortier, 1998), nous avons tenté de répondre à la question suivante: Pour comprendre et interpréter adéquatement le texte didactique de base (TDB) et le paratexte (documents historiques et iconographiques, questionnaires d'autoévaluation, glossaire, etc.), quelles compétences particulières les élèves doiventils mobiliser?

Dans ce but, nous avons étudié le chapitre consacré à l'Empire romain dans les deux manuels d'histoire les plus utilisés au Québec de 1994-1995 à 2004-2005 dans les classes d'histoire de $2^{\mathrm{e}}$ secondaire ( $8^{\mathrm{e}}$ année de scolarité, élèves de 13 et 14 ans). Il s'agit des manuels suivants: 1) De la préhistoire au siècle actuel (Dauphinais, 
1994, p. 124-158), appelé M1, et 2) Enjeux et découvertes (Roby et Paradis, 1995, p. 150-179), appelé M2.

Après avoir présenté les assises théoriques de l'étude, nous analyserons les éléments communs à tous les chapitres: la table des matières, le glossaire et l'index, puis les composantes de chaque chapitre et leur structure. Nous tenterons de cerner l'effet des choix opérés par les concepteurs des manuels sur la compréhension et l'interprétation du chapitre par l'élève-lecteur. Notons que ces concepts seront définis plus loin.

Précisons d'emblée qu'une étude interne de manuels de ce type ne porte pas sur les contenus disciplinaires traités: il ne s'agit ni d'une analyse de contenus ni d'une analyse de discours - ce qui ne relève pas de nos compétences -, mais uniquement d'une étude sur la lisibilité des manuels pour leur destinataire (Bourbeau, 1988; Bronckart et Revaz, 1988; Dery et Vidal, 1994). En effet, bien que les auteurs s'adressent directement aux élèves et qu'ils conçoivent les manuels comme des substituts du travail enseignant (Lebrun, 2006), on ne peut ignorer le fait qu'ils comptent sur l'intervention des enseignants, pour lesquels ils produisent d'ailleurs de volumineux guides.

\section{Les fondements de l'étude}

À l'école, aujourd'hui comme hier, les manuels sont des médiateurs essentiels des savoirs et savoir-faire disciplinaires (Lebrun, 2006). Aussi est-il important d'évaluer quelles compétences leur compréhension et l'interprétation mobilisent et dans quelles conditions ils peuvent jouer efficacement le rôle que l'institution (programmes, éditeur scolaire, corps enseignant) leur attribue, et ce, même si, selon Vargas (2006), ils sont, par nature, porteurs d'imperfections inhérentes et contingentes.

Les pratiques d'enseignement-apprentissage au secondaire (élèves de 12 à 17 ans) sont en grande partie constituées par la transmission-réception d'informations qui ont le statut de savoirs institutionnalisés (Blaser, 2007). Si l'interaction orale occupe un espace important dans la dynamique de la classe, la part dévolue à l'écrit (à la lecture et à l'écriture) est, elle aussi, appréciable, selon les observations réalisées dans le cadre de la recherche Scriptura dans les classes de sciences et d'histoire. L'enseignant et le manuel, sur lequel il appuie une partie significative de son enseignement, sont les principaux médiateurs des connaissances disciplinaires scolaires. Principaux agents de l'institutionnalisation du savoir scolaire, ils concourent à sa légitimité.

\subsection{Relations entre langage écrit, conceptualisation et apprentissage}

Depuis une décennie, dans la perspective ouverte par les travaux de Vygotski (1997) et de Goody (1979), la didactique du français étudie les relations entre langage écrit, conceptualisation et construction des connaissances disciplinaires scolaires, postulant qu'à l'instar des champs de recherche, chaque discipline scolaire produit 
ses propres genres textuels (Bernié, 2006; Chabanne et Bucheton, 2002; Schneuwly, 1995). Cela a amené les didacticiens des sciences à étudier les traits particuliers ou les caractéristiques des écrits scolaires en sciences (Astolfi, 1986, 1987; Fillon et Vérin, 2001), mais, curieusement, le travail a peu été fait en histoire (Moniot, 1993). Sans doute le discours historique, lorsqu'il est destiné aux jeunes lecteurs, est-il perçu comme peu spécifique. Nous verrons qu'il présente pourtant de nombreux traits spécifiques et que, contrairement aux idées reçues, ce n'est pas parce qu'un élève arrive à comprendre, sans trop de mal, les textes imposés en français qu'il peut lire avec aisance les textes des autres disciplines. Voir les choses autrement serait se méprendre sur les connaissances et les habiletés en jeu dans la compréhension en lecture, en particulier sur le rôle du contexte.

\subsection{Lire, comprendre et interpréter}

Cependant, avant d'aller plus loin, il est nécessaire de préciser ce qu'on entend par lecture, compréhension, interprétation et compétence en lecture. Comme le soulignent Morais, Pierre et Kolinsky (2003, p. 52), il apparaît qu'on doit distinguer lecture, compréhension et interprétation de textes, car faute de faire cette distinction fondamentale [...], on fonde des approches d'enseignement [de la lecture] sur des modèles de lecteurs adultes compétents. Selon ces chercheurs en sciences cognitives, [les] processus spécifiques de la lecture sont les processus perceptifs et cognitifs qui permettent de faire en sorte que la forme physique du signal graphique ne constitue plus un obstacle à la compréhension du message écrit (Morais, Pierre et Kolinsky, 2003, p. 53). Mais si lire, c'est cela, qu'est-ce que comprendre un texte? Et encore, qu'est-ce que l'interpréter? S'agit-il de deux processus distincts? Le débat est ouvert depuis longtemps, même si on s'entend pour dire que les lecteurs compétents mènent les deux processus de façon concomitante (Falardeau, 2003).

Pour "comprendre", l'on doit s'écarter de la microstructure lexicale et syntaxique pour réorganiser les informations dans une structure globalisante, qui rende intelligibles les informations essentielles du contenu du texte. Cette généralisation cherche à dégager un "sens", mot qui se définit étymologiquement comme une "perception», une représentation d'ensemble qu'actualise le lecteur à l'aide de ses connaissances dans un discours essentiellement paraphrastique. Le sens perçu participe à un certain consensus et, s'il est conditionné socialement, n'exige pas nécessairement une mise en discours, une confrontation sociale pour être reconnu.

Pour «interpréter", le lecteur ausculte le texte de manière attentive pour explorer les récurrences et déployer un des possibles signifiants. Ce n'est plus le sens qu'il poursuivra mais une "signification", dont l'étymologie renvoie directement à l' "action d'indiquer». La lecture devient ainsi actualisation sociale d'un signe créé; elle n'est plus seulement représentation personnelle, puisqu'elle doit nécessairement passer par la confrontation sociale pour acquérir une certaine légitimité. Le texte polysémique se transforme alors en matériau d'un nouveau texte - l'interprétant peircien -, fruit de la création du lecteur qui déborde du texte original (Falardeau, 2003, p. 675). 
Dans un mouvement itératif, le lecteur s'éloigne du texte-compréhension-et s'en rapproche-interprétation - pour constamment changer sa perspective et l'adapter à la fois à ses connaissances et aux signes tirés du texte (p. 675). Ainsi il n'y aurait pas de différence de degré ou d'antériorité obligée de l'un par rapport à l'autre, il s'agirait plutôt d'une

[...] tension dynamique et obligée entre d'une part la vision macroscopique, qui part du texte pour s'en éloigner et, d'autre part, la vision microscopique, qui part de l'extérieur du texte pour s'y plonger et y explorer des microstructures récurrentes - microstructures qui peuvent irradier sur l'ensemble du texte (Falardeau, 2003, p. 678).

Cependant, ajoute Falardeau (2003), s'appuyant sur des didacticiens du texte littéraire, il serait naïf de croire que les élèves du secondaire et du collégial possèdent toutes les compétences de compréhension et d'interprétation des textes qu'ils doivent lire. Ils sont encore en apprentissage et le rôle de l'école, pas seulement de l'enseignant de français, est de développer leurs compétences lectoriales. Par compétence, nous entendons un ensemble dynamique de connaissances, d'habiletés et d'attitudes qui peut être mobilisé pour réaliser efficacement une tâche. Comment développer ces compétences lectoriales?

\subsection{Acquis des recherches sur la lecture}

Les recherches sur les compétences en lecture des novices comme des experts montrent la très grande complexité des processus de compréhension et d'interprétation en lecture. On s'entend pour dire que lire est une activité langagière et cognitive qui fait intervenir des processus mentaux de bas et de haut niveau. Sommairement, la lecture est une activité dynamique qui met en jeu trois composantes interreliées: le lecteur (ses capacités cognitives, son affectivité, son engagement et ses dispositions devant un projet ou une tâche de lecture, ses expériences de lecture, son milieu socioculturel, sa connaissance du monde, de la langue et des modèles de textes), le texte (thème, particularités du genre textuel, caractéristiques linguistiques, rhétoriques, graphiques) et le contexte de lecture (cadre spatiotemporel de l'activité, projet ou tâche de lecture, lieu social de réception). Plus l'interaction entre les trois composantes est forte, plus la compréhension est bonne (Giasson, 2000). Première conséquence didactique de ce modèle, il n'y a pas, dans l'abstrait, de bons ou de mauvais lecteurs: il n'y a que des lecteurs plus ou moins compétents selon le genre de texte, le contexte et la tâche ou le projet de lecture. Deuxième conséquence, tout individu peut développer ses compétences en lecture tout au long de sa vie. Bref, on ne sait pas lire une fois pour toutes à la fin de la scolarité obligatoire. Troisième conséquence: le fait que la capacité à comprendre dépend du contexte et du genre de texte entraîne que le seul cours de français ne permet pas à tous les élèves de se familiariser avec les textes qu'ils devront lire dans toutes les disciplines scolaires et, donc, ne les amène pas à acquérir les outils sémiotiques spécifiques pour comprendre les différents genres disciplinaires (Castellani, 1995). Dès lors, si l'on juge important que les élèves aient accès à une 
compréhension fine des contenus des manuels disciplinaires, ils doivent y être initiés par l'enseignant de chaque discipline. C'est ce que reconnaissait Moniot (1993), qui soutenait qu'il faut entraîner les élèves à lire les divers textes à visée didactique en histoire pour qu'ils reconnaissent et apprécient les ingrédients et les mises en relation constitutives du discours didactique (p. 192).

Les sciences cognitives ont élaboré un modèle général qui rend compte du processus de compréhension de textes chez les lecteurs compétents, fournissant ainsi des pistes pour l'enseignement de la compréhension des textes. Il y aurait trois composantes interreliées dynamiquement et soumises à un contrôle cognitif: la planification de la lecture (se donner une intention de lecture, survoler le texte, faire émerger ses connaissances sur le thème, le genre lu, faire des prédictions, etc.), la (re)construction du sens et de la signification (activer des connaissances sur le monde, les genres et la langue écrite, mettre en relation des éléments, produire des inférences, etc.) et la réaction émotive et intellectuelle (évaluer, critiquer, élaborer, etc.). Aussi, la compréhension et l'interprétation des textes mettent en branle des activités mentales tout au long du processus de lecture; c'est pourquoi, selon un modèle didactique répandu, on propose d'intervenir avant, pendant et après la lecture, afin de soutenir la compréhension des textes chez les apprentis lecteurs que sont les élèves du secondaire (Giasson, 1995a).

\section{Méthodologie: une analyse interne à trois niveaux}

L'analyse du corpus a été menée à trois niveaux: le premier se rapporte à la structure des chapitres: leurs composantes, la hiérarchisation des contenus et les interactions des diverses composantes avec les outils généraux du manuel (la table des matières, le glossaire et l'index); le deuxième appréhende les phénomènes textuels proprement dits: les séquences textuelles (description, narration, explication, argumentation), les procédés discursifs (définition, exemplification, langage figuré, etc.), ainsi que la cohérence textuelle (système anaphorique et énonciatif); enfin, le troisième vise à étudier les particularités phrastiques et lexicales: structures syntaxiques privilégiées, rôle sémantique de la ponctuation (deux-points, tiret, parenthèse), particularités du vocabulaire (spécialisé ou courant).

Ces trois niveaux d'analyse sont nécessaires, compte tenu que la compréhension et l'interprétation du manuel, discours hybride et complexe, se construisent sur ces différents niveaux. Cependant, pour des raisons d'espace, nous ne rendrons compte ici que de l'analyse exhaustive du premier niveau, nous limitant à faire des remarques ponctuelles sur les deux autres.

Le premier niveau concerne la structure des chapitres: leurs différentes composantes, leurs fonctions et leurs liens explicites ou implicites, car pour connaitre quelles formes prend la mise en discours dans un manuel, il faut appréhender ensemble toutes ses composantes, telles que les marques typographiques, les modes de mise en discours du texte didactique de base (TDB), les documents didactiques (questions, autoévaluations, présentation des objectifs d'apprentissage), historiques 
(reproduction d'un texte de source première) et iconographiques, car elles participent toutes à la construction du sens lors de la lecture et de l'étude (GinsburgerVogel, 1988).

\section{Résultats}

\subsection{Les éléments communs aux chapitres: la table des matières, le glossaire et l'index}

Pour analyser les défis liés à la compréhension d'un chapitre, on doit considérer les outils tels que la table des matières, le glossaire et l'index, car ce sont des repères qui permettent à l'élève de tirer profit de son manuel (Gérard et Roegiers, 1993). Bien qu'indépendants des chapitres, ces outils facilitent ou entravent, à différents moments, la compréhension du texte.

\subsubsection{La table des matières}

La table des matières d'un manuel a trois fonctions principales: elle permet au lecteur 1) d'avoir un aperçu du contenu thématique de l'ouvrage (ce qu'on appelle la macrostructure sémantique);2) de saisir son organisation interne, celle des chapitres ainsi que la place du chapitre étudié dans l'ensemble de l'ouvrage, et 3) d'aider à sa consultation. Cependant, pour jouer pleinement son rôle d'aide à la compréhension, elle doit présenter certaines caractéristiques: précision des entrées sémantiques et présentation de l'organisation séquentielle et hiérarchique des contenus par la mise en page et les choix typographiques, notamment. Dans ces conditions, elle constitue un outil qui facilite l'intégration des contenus principaux du manuel dans une représentation globale du texte (Bourbeau, 1988). Comparons la façon dont sont présentées les tables des matières des chapitres analysés.

\begin{tabular}{|c|c|c|}
\hline \multicolumn{2}{|c|}{ Chapitre 6. Grandeur et déclin de la cité de Rome } & 124 \\
\hline Section A & $\begin{array}{l}\text { L'expansion finale et le maintien de l'Empire de Rome } \\
\text { 1. La puissance de l'armée et le pouvoir impérial } \\
\text { 2. Un réseau de communication } \\
\text { 3. Des impôts considérables versés en monnaie métallique } \\
\text { 4. Le travail des populations soumises } \\
\text { 5. Les femmes dans la Rome impériale des } 1^{\text {er }} \text { et } 2^{\mathrm{e}} \text { siècles }\end{array}$ & $\begin{array}{l}128 \\
128 \\
131 \\
134 \\
136 \\
138\end{array}$ \\
\hline Section $\mathbf{B}$ & $\begin{array}{l}\text { La vie à Rome après les conquêtes } \\
\text { 1. Une masse de citoyens entretenus et oisifs } \\
\text { 2. Des jeux pour occuper les loisirs }\end{array}$ & $\begin{array}{l}140 \\
141 \\
142\end{array}$ \\
\hline Section $\mathbf{C}$ & $\begin{array}{l}\text { Le déclin de l'Empire en Occident } \\
\text { 1. La montée du christianisme } \\
\text { 2. L'affaiblissement du pouvoir impérial } \\
\text { 3. La poussée finale des tribus germaniques }\end{array}$ & $\begin{array}{l}145 \\
145 \\
151 \\
153\end{array}$ \\
\hline En résumé & & 156 \\
\hline
\end{tabular}

Figure 1. Table des matières du chapitre 6 de $\mathrm{M} 1$, De la préhistoire au siècle actuel 
La table des matières de M1 (Figure 1) donne facilement accès à la macrostructure du chapitre. Le lecteur, grâce à la présence de marques typographiques (espaces, identification des sections par un titre et une lettre, numérotation des sous-sections), peut se rendre compte que le chapitre comporte trois parties divisées elles-mêmes en sous-parties. Cette mise en relief de l'organisation interne facilite la lecture et l'étude, voire la mémorisation des contenus du chapitre, puisqu'elle permet à la fois la création d'une représentation globale et hiérarchique des contenus du texte et dégage les idées principales, deux conditions essentielles à toute compréhension (Dumortier, 1999). Qu'en est-il de M2 (Figure 2)?

\section{Chapitre 7: La société de l'Empire romain}

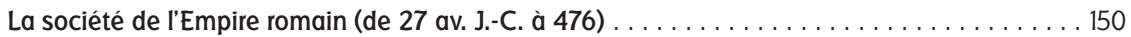

Des idées à creuser : Qu'est-ce qu'un empire? . . . . . . . . . . . . . . . . . . . . . 152

Sur les traces de...: Caius Julius Cæesar, dit « Jules César » (101 à 44 av. J.-C.) . . . . . . . . . . . 153

La puissance de Rome ou les bases de l'expansion et du maintien de l'Empire romain . . . . . . . . 154

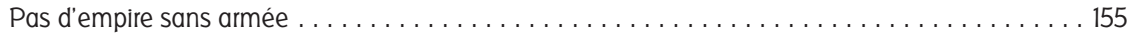

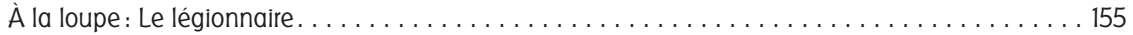

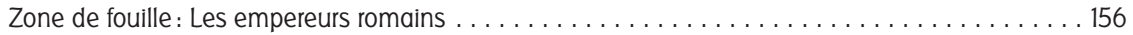

Les pouvoirs de l'Empereur . . . . . . . . . . . . . . . . . . . . . . . . . . . . . . 159

Sur les traces de : Livia Drusilla dite "Livie» (58 av. J.-C. à 29) . . . . . . . . . . . . . . . . 161

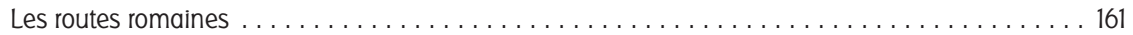

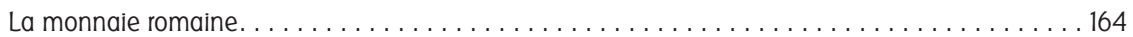

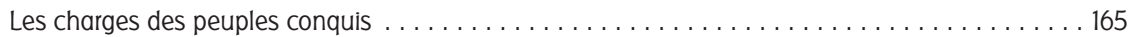

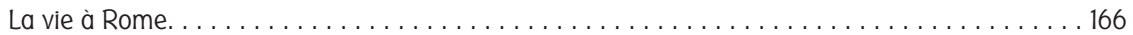

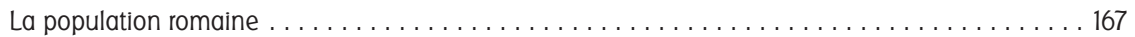

Panem et circenses. . . . . . . . . . . . . . . . . . . . . . . . . . . . 168

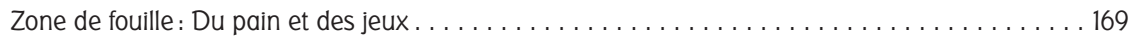

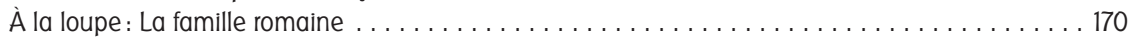

La décadence de Rome ou les signes du déclin et de la chute de l'Empire romain d'Occident . . . 171

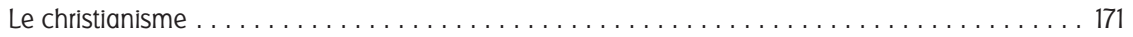

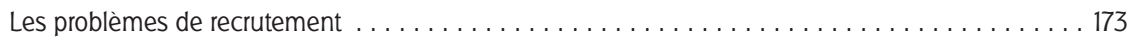

Les difficultés économiques . . . . . . . . . . . . . . . . . . . . . . . . . . 174

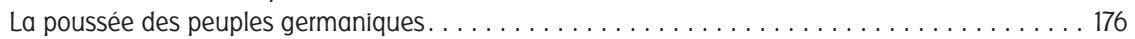

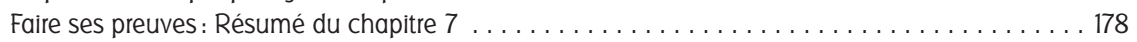

Figure 2. Table des matières du chapitre 7 de M2, Enjeux et découvertes

À l'inverse, la table des matières de M2 (Figure 2) ne permet pas la création d'un tel schéma mental, puisqu'elle ne présente aucune structuration interne des contenus, rien n'étant mis en relief. En effet, les 23 parties du chapitre sont présentées sous la forme d'une liste, sans être numérotées ni hiérarchisées. Bien qu'ils existent, les liens entre les titres ne sont pas marqués. Prenons, par exemple, les cinq titres suivants: 1) La décadence de Rome ou les signes du déclin et de la chute de l'Empire romain d'Occident; 2) Le christianisme; 3) Les problèmes de recrutement; 4) Les difficultés économiques et 5) La poussée des peuples germaniques. Ces titres sont au même niveau, donc ils semblent de même importance. Pourtant, dans le texte didactique de base, un lecteur aguerri comprend que le premier titre ( $\mathrm{La}$ 
décadence...) domine les quatre autres, qui sont des illustrations ou des explications de la décadence de l'Empire. Un tel exemple d'une caractéristique de la mise en discours dans ces manuels permet de constater que jamais les relations de cause à effet et, plus globalement, les explications des phénomènes ne sont mises en évidence; c'est au lecteur de déceler les explications à l'intérieur des séquences descriptives. Cette caractéristique des textes dans les manuels d'histoire a été aussi observée par Laparra (1991).

Force est de constater que cette disposition n'aide pas le jeune lecteur à se faire une représentation adéquate de ces contenus et ne lui permet ni d'anticiper les contenus ni de comprendre leur articulation. Il devra faire ce travail lui-même. Cependant, cette table des matières comporte des particularités intéressantes: par exemple, l'utilisation de lettres colorées qui fait ressortir la présence des différentes rubriques (Des idées à creuser, Zone de fouilles...). Ces rubriques, qui se démarquent du texte didactique de base, abordent l'histoire sous différents aspects: elles présentent des concepts essentiels, des documents historiques, des personnages marquants, etc. En différenciant les rubriques des différentes parties du texte didactique de base, le lecteur est amené à prendre connaissance de l'objectif précis de chacune d'elles, ce qui lui permet de circonscrire son horizon d'attente et de préciser ses intentions de lecture pour chacune. En ce sens, la mise en relief du titre des différentes rubriques constitue une aide à la lecture, en dépit des difficultés que peut représenter le vocabulaire dans ces tables des matières pour des élèves de $2^{\mathrm{e}}$ secondaire (pouvoir impérial, tribus germaniques, charges des peuples conquis, etc.).

Ajoutons qu'une table des matières ne peut servir d'outil à la compréhension que si elle est consultée. Or, la majorité des élèves de $2^{e}$ secondaire n'ont pas l'habitude de consulter une table des matières, n'en voyant pas la nécessité. Il importerait donc de leur montrer, par un enseignement explicite, la pertinence de cet outil et de leur faire acquérir les habiletés méthodologiques pour rendre son utilisation efficace.

\subsubsection{Le glossaire}

Le glossaire a deux fonctions. L'une est explicite: donner le sens du mot, compte tenu du cotexte; l'autre, implicite: souligner la spécificité des mots du texte didactique de base, marquant leur appartenance à la discipline, ce qui peut amener les élèves à développer une conscience disciplinaire, et notamment à saisir que chaque discipline possède son vocabulaire. Comme cette prise de conscience n'est pas automatique, elle demande toutefois un étayage de la part de l'enseignant.

Par nature, un manuel contient des contenus, des notions et des concepts nouveaux, inconnus des élèves. L'appropriation de nouveaux contenus disciplinaires peut difficilement se faire sans l'utilisation d'un vocabulaire spécialisé (Ministère de l'Éducation nationale, 1997). Le glossaire placé à la fin du manuel constitue pour l'élève un moyen d'accéder au sens des mots nouveaux (monothéisme, empire, centurie) ou des mots dont le sens est particulier au domaine d'études (domination, 
révolte, hiérarchie). La compréhension du texte didactique de base dépend donc des compétences lexicales des élèves et de l'utilisation qu'ils font de stratégies adéquates pour la compréhension de mots et de sens nouveaux (utilisation du cotexte, d'indices grammaticaux et lexicaux: la dérivation par exemple et le recours à des ouvrages terminologiques). Dans un manuel, un des avantages du glossaire, si on compare celui-ci au dictionnaire de langue, est qu'il comporte des définitions relevant du domaine d'études dont la formulation est adaptée aux élèves. Il s'agit d'un outil essentiel à la compréhension d'un texte disciplinaire, car de l'avis des enseignants sondés dans l'enquête Scriptura, les principaux problèmes de compréhension des textes à lire en histoire sont d'ordre lexical (définition, champs sémantiques et lexicaux), ce qu'a aussi constaté Laparra (1991). Mais le glossaire aide-t-il vraiment?

La consultation efficace du glossaire demande des habiletés qui requièrent un entraînement. Une fois identifié le mot faisant l'objet d'un renvoi au glossaire (dans les deux manuels, le renvoi se fait par l'écriture du mot en gras), l'élève doit arrêter sa lecture, chercher le glossaire, puis le mot à l'intérieur de ce dernier, lire la définition et la comprendre, puis retourner au texte, actualiser et vérifier sa compréhension. Toutefois, la lenteur de la manipulation et de l'investigation ainsi que tous les «oublis» qu'elle occasionne (oubli du mot, de sa définition, du contexte) risquent [...] d'entraver la compréhension par le morcèlement et le fractionnement qu'elle induit (Ginsburger-Vogel, 1988, p. 14). La compréhension n'est pas nécessairement assurée par ce parcours. L'élève doit donc disposer de stratégies lorsqu'il rencontre un mot dans le glossaire et savoir laquelle utiliser pour chaque cas: soit arrêter la lecture et aller consulter le glossaire (avec les risques que cela comporte); soit, en se servant d'indices du contexte, tenter de se faire une idée du mot, quitte à aller consulter le glossaire à la fin de la phrase ou du paragraphe, dans le but de confirmer son hypothèse ou de la préciser. Faut-il souligner que la compréhension de mots nouveaux grâce au glossaire n'entraîne pas nécessairement leur rétention et la possibilité de leur utilisation en contexte?

\subsubsection{L'index}

L'index a pour fonction de faciliter l'accès au texte lors de la recherche d'un contenu ou d'un concept et de mettre en lumière la richesse informative d'un ouvrage. Par contre, une consultation efficace nécessite, elle aussi, un savoir méthodologique que la majorité des élèves n'ont pas acquis (Ginsburger-Vogel, 1987, p. 55) et qu'il convient d'enseigner.

L'index de M1 est intégré au glossaire, les auteurs ayant sans doute misé sur la complémentarité des deux outils afin d'en simplifier l'utilisation. Lorsqu'il consulte le glossaire-index du manuel, l'élève a accès à la définition du mot en même temps qu'à un renvoi vers le texte. Cette combinaison facilite la compréhension des notions du glossaire en réduisant les étapes lors de la recherche, car M1 ne donne qu'un renvoi par mot. Cependant, elle limite de beaucoup la richesse informative 
de l'outil. En effet, si l'on compare les deux manuels, on constate que le glossaireindex de M1 contient 147 entrées, alors que l'index de M2, indépendant du glossaire, en contient 631 ! De plus, M2 donne jusqu'à cinq renvois par mot. Néanmoins, en regard des besoins et des compétences lexicales et disciplinaires des élèves de $2^{\mathrm{e}}$ secondaire, on peut douter que ceux-ci soient en mesure d'exploiter pleinement la richesse informative d'un index comme celui de M2.

Il ressort de ce qui précède que plusieurs conditions doivent être remplies pour que la table des matières, l'index et le glossaire deviennent de puissants outils non seulement pour la consultation du manuel, mais aussi pour la compréhension de ses contenus. Cependant, la compétence à utiliser ces outils n'est pas développée d'emblée chez de jeunes lecteurs et elle ne saurait l'être pour la majorité d'entre eux sans un enseignement explicite et un accompagnement périodique de l'enseignant (Aeby, 2004; Soussi, 1995).

\subsection{Les composantes d'un chapitre et leurs fonctions}

Les chapitres analysés ont chacun six composantes, dont trois sont des auxiliaires à l'apprentissage: les objectifs d'apprentissage, les questions et les autoévaluations, et un résumé du chapitre; et trois concernent les contenus disciplinaires: le texte didactique de base, les documents historiques et iconographiques (et leur légende à l'occasion).

\subsubsection{Les objectifs d'apprentissage}

Chaque chapitre de M2 commence avec la présentation d'une liste d'objectifs d'apprentissage tirés du Programme d'études (Ministère de l'Éducation du Québec, 1982). De façon explicite, à l'aide d'une interpellation (à la fin de ce chapitre, $t u$ devrais être en mesure...), l'auteur s'adresse directement à l'élève en lui faisant part des connaissances qu'il devra développer par la lecture du chapitre. Cette composante agit comme une aide à la compréhension en lecture: elle [...] permet [au lecteur] de créer des expectatives qui sont [de] véritable[s] motivation[s] interne[s] pour apprendre (Gérard et Roegiers, 1993, p. 52). Elle favorise la création d'une intention de lecture et la recherche des informations les plus importantes au cours de la lecture. Cependant, bien qu'utile, la présence d'objectifs d'apprentissage ne suffit pas pour que l'élève se dote d'une intention de lecture: l'enseignant doit intervenir pour en montrer l'importance et présenter différentes stratégies de planification de la lecture (survoler le texte, faire des prédictions, consulter la table des matières du chapitre, etc.). Ainsi, l'élève pourra concevoir un projet de lecture, se créer une représentation initiale des attentes et du sujet (en faisant des prédictions à l'aide des titres, des sous-titres, des illustrations, etc.) et activer ses connaissances sur celui-ci.

Dans M1, les objectifs d'apprentissage sont moins visibles et moins explicites que dans M2. Au lieu d'apparaître en bloc, ceux-ci sont présentés un à un, au début du chapitre ainsi qu'au début de chacune des trois sections principales. En outre, il faut 
inférer que ces énoncés à l'infinitif sont des objectifs d'apprentissage, ce qui ne va pas nécessairement de soi. Ce choix, si l'on suppose que l'élève a compris le sens de ces énoncés, a lui aussi ses avantages; il permet à l'élève de se fixer des objectifs progressivement, au commencement de chacune des grandes parties du chapitre.

\subsubsection{Les questions des autoévaluations}

Les questions posées dans la section autoévaluation et dans l'ensemble du chapitre sont un indicateur du niveau de compréhension attendu des élèves et des compétences interprétatives qu'ils doivent déployer. D'après le modèle de Cunningham (Giasson, 1995a, p. 60), pour susciter la compréhension ou pour l'évaluer, deux types de questions peuvent être posées: les premières sont littérales; les secondes, inférentielles. Compétence de bas niveau sur le plan cognitif, la compréhension littérale est celle qui est sollicitée lorsque les questions portent sur la microstructure du texte (un segment de phrase, une phrase ou deux) et que les réponses se trouvent directement dans le texte, qu'elles sont explicites et sémantiquement équivalentes ou synonymes d'une partie de texte (Giasson, 1995a, p. 61). Pour répondre à une question implicite, l'élève n'a qu'à comprendre la question et à repérer, grâce à des indices linguistiques (généralement lexicaux), la réponse dans le texte. Sa capacité à comprendre les liens explicites et ses connaissances lexicales vont déterminer la qualité de sa compréhension littérale du texte. La compréhension inférentielle, par contre, mène le lecteur à une compréhension de haut niveau, l'obligeant à aller plus loin que ce qui est présent à la surface du texte, à découvrir l'implicite discursif en faisant appel à ses connaissances du sujet et des textes, et à établir des liens entre les différentes composantes informatives du texte. Pour répondre à la question posée, le lecteur doit, par son raisonnement, saisir un rapport implicite dans le texte. Or, c'est une habileté de haut niveau, par conséquent peu maîtrisée chez des élèves de cet âge (Soussi, 1995).

À l'aide du modèle de Cunningham (Giasson, 1995a), nous avons effectué une analyse systématique des questions des autoévaluations. La majorité des questions font appel à une compréhension littérale, visant donc le repérage de l'information, sans que celle-ci soit nécessairement comprise et encore moins interprétée. La plupart des questions restent tellement collées au texte qu'elles n'amènent pas l'élève à saisir le motif central qui s'en dégage. Seulement $41 \%$ des 43 questions dans M2 et $35 \%$ des 80 questions dans M1 sont inférentielles et impliquent un processus de traitement et d'interprétation de l'information. Enfin, soulignons que dans M2, un nombre non négligeable de questions ( 5 sur 43), appelant des réponses factuelles, ne peuvent trouver de réponses à l'aide du manuel! Par exemple, dans M2 (p. 153), on pose une question en termes d'années et il n'est possible, avec les informations disponibles dans le texte, de répondre qu'en termes de siècles.

Ici, nous faisons face à un paradoxe, car, comme nous le verrons, la compréhension et l'interprétation du texte didactique de base et des documents exigent des compétences de lecture complexes de la part des élèves, alors que les questions 
d'autoévaluation, qui doivent mesurer l'atteinte des objectifs d'apprentissage, elles, font majoritairement appel à des compétences de bas niveau.

\subsubsection{Le résumé}

Le résumé de trois pages de $\mathrm{M} 1$ prend la forme d'une liste schématique qui intègre tous les titres et sous-titres du chapitre, ce qui permet à l'élève qui le consulte de renforcer sa connaissance de la macrostructure sémantique du chapitre. Cependant, comme dans le texte didactique de base, les liens entre les parties sont généralement implicites (quel est le sens des flèches entre deux items: causalité, temporalité, inclusion?). Il y a un seul endroit où on indique que des facteurs interreliés expliquent l'expansion et le maintien de l'empire de Rome (Dauphinais, 1994, p. 156). Dans M2, dans la page Résumé (Roby et Paradis, 1995, p. 178), on ne trouve pas de résumé proprement dit, mais une liste de mots à insérer dans un organigramme. Cela constitue une tâche exigeante, mais elle facilite l'apprentissage, car les relations d'inclusion sont manifestes. On peut cependant douter que la majorité des élèves parviennent seuls à la réaliser, mais elle peut être l'occasion d'une explicitation collective des liens entre les informations.

\subsubsection{Le texte didactique de base}

Dans les deux manuels, le texte didactique de base, bien qu'il représente une portion modeste du chapitre, en constitue le fil conducteur. Il s'agit d'un texte essentiellement descriptif, dont le plan est fortement structuré: les titres et les sous-titres, qui sont des marques de cette structuration (tout comme les blancs et les paragraphes), agissent comme des indicateurs thématiques importants. En effet, [ils reflètent] l'essentiel de l'information ou l'idée directrice d'une séquence et permettent ainsi au lecteur de saisir la logique existant entre les différentes parties du chapitre (Bouchard et Tessier, 1994, p. 45). En plus d'envisager les titres comme des indicateurs thématiques, l'élève doit percevoir et interpréter les différents indices typographiques qui marquent une hiérarchie entre les titres et sous-titres (les variations typographiques les plus fréquemment utilisées étant le caractère gras, la taille des polices, les majuscules, etc.) et saisir la relation de sens entre les différentes parties et leurs sous-parties. Selon Giasson (1995a), la compréhension de la structure de l'exposé didactique d'un manuel à l'aide des titres et des sous-titres demande un savoir-faire complexe, qui semble peu maitrisé par la majorité des élèves de $2^{\mathrm{e}}$ secondaire (Aeby, 2004; Giasson, 1995a; Ministère de l'Éducation nationale, 1997; Soussi, 1995).

Ces relations peuvent être de type temporel ou causal, ou encore inclusif (du tout à la partie) et leur interprétation relève de l'implicite textuel, ce qui est très exigeant sur le plan cognitif et implique des compétences de lecture de haut niveau (Ministère de l'Éducation nationale, 1997; Sylvanise, 1993). Par exemple, dans M1, il n'y a aucune séquence explicative: les relations causales entre deux phénomènes exposés doivent être déduites, car aucun indice linguistique ne les révèle. 
Pourtant, la compréhension de ces relations est nécessaire à une représentation articulée et logique des différentes informations principales du texte didactique de base, donc de la macrostructure du texte. Cependant, elle est rendue difficile à cause de l'importance des illustrations qui fait que le texte didactique de base est présenté par petits segments. De page en page, le lecteur n'a accès qu'à une mince part du texte didactique. La vue d'ensemble d'une grande partie de chapitre, lors d'une première lecture, n'est donc pas possible. Ainsi, la macrostructure du texte, qui donne accès au sens et à l'orientation globale du texte (Dumortier, 1999), est difficile à dégager; l'anticipation est, par conséquent, ardue. L'organisation des informations en une représentation cohérente ainsi que leur éventuelle mémorisation s'en voient donc entravées (Giasson, 1995a; Soussi, 1995).

Autre caractéristique du texte didactique de base, le système énonciatif utilisé. On privilégie un contrat de communication où l'auteur entre directement en relation avec le lecteur par diverses marques d'interpellation ( $t u$, te, toi) et de complicité énonciative (nous = toi et moi), pour réduire la distance entre celui qui sait et celui qui doit apprendre; ainsi le texte didactique de base se présente comme le produit de la grande voix de l'Histoire, et non comme une énonciation parmi d'autres. Ici, l'histoire se présente comme un pur énoncé [...]. Le discours historien de l'école, comme tout discours pédagogique, est un discours injonctif, qui dicte au nom du réel ce qu'il faut dire ou croire (René, 1991, p. 71). Nulle marque de modélisation du discours qui pourrait signaler une opinion, un jugement ou une incitation à prendre position. Cela renforce l'impression que ce qu'il y a d'important dans le texte, ce sont les informations factuelles, que c'est de cela qu'est faite l'histoire.

\subsubsection{Les documents historiques et iconographiques}

$\mathrm{M} 1$ et M2 contiennent chacun cinq documents historiques, des citations d'environ 60 mots d'auteurs romains, d'historiens ou de dirigeants politiques (Sénèque, Tacite, Pline le Jeune, César). Aucun n'est commenté, trois sont très brièvement introduits et contextualisés. S'ils ne présentent pas de difficultés de compréhension littérale, on imagine mal comment les élèves peuvent les interpréter, vu le manque de contextualisation des sources et des auteurs.

Les documents iconographiques, eux, sont fort variés et présentent des marques typographiques particulières (trame ou caractères de couleur), ce qui permet de bien les distinguer du texte didactique de base. Il s'agit de photos d'objets ou de monuments historiques (sculptures, mosaïques, routes, murailles, arches, pièce de monnaie, etc.), de dessins originaux et de représentations graphiques (schémas, tableaux, diagrammes, cartes géographiques, ligne du temps, histogramme, etc.). Ils occupent l'essentiel de l'espace: dans M1, ils occupent les deux tiers de l'espace et dans M2, les trois quarts du chapitre, ce qui occasionne une mise en page surchargée, où les informations écrites et iconographiques ne sont ni hiérarchisées ni organisées. Dans M2, 23 documents numérotés sont présentés à l'élève; un peu 
plus de la moitié sont accompagnés de questions et les deux tiers, d'un court texte qui commente ou explique le texte didactique de base.

Les documents assument ou assurent plusieurs fonctions: 1) une fonction cognitive: aider à se bâtir une image mentale ou activer la mémoire visuelle pour apprendre; 2) une fonction informative: authentifier une information du texte didactique de base, ajouter de l'information ou l'exemplifier; 3) une fonction incitative: piquer la curiosité, susciter l'intérêt et la motivation pour la lecture; 4) une fonction esthétique et d'éducation culturelle: faire découvrir des œuvres d'art; et 5) une fonction pragmatique: économiser de l'espace. Notons que ces fonctions ne sont pas exclusives: une même illustration peut en avoir plus d'une. Dans les manuels analysés, la majorité des illustrations ont des fins informatives, bien que la pertinence de l'information fournie ne soit pas toujours évidente en regard du texte didactique de base, surtout quand le document n'est pas commenté, ce qui est souvent le cas (pour les dessins, notamment) ou quand le commentaire semble peu adéquat pour l'interprétation du document, ce qui n'est pas rare. Par exemple, à propos du Document 3.35, commenté plus loin, deux questions laissent pantois, car il est impossible d'y répondre sans avoir des connaissances en construction navale, et encore...

Or, la compréhension et l'interprétation des documents ne peuvent être indépendantes du texte didactique de base: le texte limite la polysémie de l'image en privilégiant une lecture parmi plusieurs possibles (Gingsburger-Vogel, 1987, p. 47). C'est donc essentiellement à partir du texte didactique de base que l'élève peut organiser la lecture des documents.

La compréhension et l'interprétation d'un document historique, que celui-ci soit écrit ou visuel, sont plus aisées lorsque le lecteur en connaît l'origine (date, lieu de production, auteur, etc.). Or, les documents d'objets historiques ne sont généralement pas accompagnés d'une mention de la source originale (sauf, bien entendu, la liste des crédits photographiques en fin de manuel, qui n'a pas, on en conviendra, d'objectif didactique): l'appartenance des objets à leur époque et même leur fonction sont, la plupart du temps, implicites, il n'y a pas de légende qui donnerait une piste d'interprétation. Comment, dans ces conditions, un élève de $2^{\mathrm{e}}$ secondaire peut-il interpréter la valeur informative, voire explicative de ces documents, avec tout ce que cela comporte (mise en relief des motivations de son ou de ses auteurs, des enjeux de sa production, établissement du lien entre une œuvre artistique et le courant dont elle fait partie, analyse des représentations et conceptions mentales qu'elle révèle, etc.)? Sans ces indications, le document est désincarné, il perd sa spécificité, sa valeur testimoniale; son intérêt esthétique et sa valeur historique en sont par conséquent diminués.

M1 contient aussi des documents variés, numérotés et toujours accompagnés de commentaires, dont la qualité ou le nombre insuffisants ne garantissent pas la valeur didactique. Prenons un exemple parmi plusieurs: le Document 3.35, dont le titre est: Le chargement d'un bateau. Il s'agit de la reproduction partielle d'une 
mosaïque d'époque (ce qui n'est pas précisé), dont le dessin naïf, aux proportions mal calculées, représente une barque munie de deux grandes rames et sur laquelle se tiennent cinq personnes (dont on peut à peine deviner l'âge ou le sexe), dont deux tiennent un sac d'une céréale quelconque et dont une est assise à l'avant. Il est accompagné de la légende suivante:

Des débardeurs chargent un bateau dans le port d'Ostie, plaque tournante du commerce de l'Empire. À l'époque, un bateau moyen, long de 20 mètres et large de 6 mètres, peut contenir de 500 à 1000 tonnes de marchandises. Entre le port d'Ostie et la ville de Rome, les marchandises sont transportées par chariot ou par petit bateau (Dauphinais, 1994, p. 133).

Il est fort difficile de faire le lien entre le document et sa légende: les petits personnages n'ont rien à voir avec la représentation que les élèves peuvent se faire de débardeurs; la barque peut difficilement être vue comme un bateau servant à une importante activité commerciale, et rien n'indique qu'on est en train de la charger. De plus, le texte didactique de base ne donne aucune indication sur le port d'Ostie, sur son emplacement par rapport à Rome et sur la nécessité de faire transiter par petits bateaux les marchandises de Rome à Ostie. À quoi sert donc cette illustration, quel lien de sens l'associe au texte didactique de base, comment l'élève peut-il la comprendre et l'interpréter?

L'imbrication des écrits (texte didactique de base, légendes, questions) et des documents iconographiques ne permet pas une lecture analogue à celle que l'on fait d'un récit ou d'un article de magazine, genres qui sont davantage familiers aux élèves. La lecture d'un manuel d'histoire, qui implique un va-et-vient permanent entre le texte et ces documents, présente bien des obstacles à sa compréhension par l'élève encore à l'âge d'apprendre à lire (Astolfi, 1987; Castellani, 1995; Darras et Delcambre, 1986; Derry et Vidal, 1994 ; Le Bouffant, 1986; Ministère de l'Éducation nationale, 1997). L'élève peut se concentrer sur le texte, et l'image est alors considérée comme une simple décoration, ou, à l'inverse, principalement sur le contenu de l'illustration, en ne faisant qu'une lecture partielle du texte didactique de base et des légendes. De plus, lecteur peu expérimenté et aux connaissances historiques embryonnaires, il est sujet à faire une interprétation personnelle des documents qui laisse beaucoup de place à sa subjectivité (cette image-ci me plaît; celle-là, non, etc.). Par conséquent, leur intérêt didactique provient de l'explicitation de leurs rapports avec le texte didactique de base. Comme les manuels ne le font généralement pas, il revient à l'enseignant de les contextualiser, de souligner leurs diverses interprétations possibles et de fournir une méthode spécifique d'analyse pour chaque type de document iconographique (Delcambre, 1988; Ministère de l'Éducation nationale, 1997; Moniot, 1993). La distanciation ainsi créée permettra à l'élève d'éviter les contresens et les mauvaises interprétations. 


\section{Conclusion}

Il ressort de cette analyse faite dans une perspective précise et limitée que, même si les auteurs s'adressent directement à l'élève ou feignent de le faire, celui-ci n'a généralement pas les compétences de compréhension et d'interprétation adéquates pour atteindre les objectifs d'apprentissage exposés dans les manuels. Paradoxalement, alors que les questions des autoévaluations, généralement littérales, ne présentent pas de défis interprétatifs, le texte didactique de base, lui, ne permet pas à la majorité des élèves de comprendre les contenus essentiels du chapitre ni d'en reconstituer la macrostructure, faute de liens explicites entre les parties, et plus globalement à cause d'une mise en discours essentiellement descriptive. Compte tenu des finalités officielles de l'enseignement de l'histoire, entre autres celle de développer l'esprit critique, il est étonnant que le texte didactique de base, par son absence de modalisation, ne permette pas à l'élève de se distancier par rapport aux contenus, qui sont présentés comme allant de soi et non comme une lecture particulière de l'histoire; ainsi, jamais l'expansion et la domination de l'Empire romain ne sont présentées de façon critique, ce qui ne permet pas non plus à l'élève de déployer une interprétation critique de l'histoire.

Conséquemment, l'enseignant qui désire que ses élèves utilisent leur manuel doit leur enseigner explicitement comment s'en servir en tant que source de données, mais aussi comme outil d'apprentissage. L'enseignement explicite de stratégies et de procédures de lecture est nécessaire, autant pour savoir exploiter les outils généraux que sont la table des matières, l'index et le glossaire, que pour faire une lecture adéquate du texte didactique de base et des très nombreux documents iconographiques, de nature fort variée, qui demandent chacun des clés spécifiques de lecture. Mais est-ce réaliste de considérer que les enseignants d'histoire ont les compétences nécessaires en didactique de la lecture pour enseigner à leurs élèves quelles stratégies et procédures de lecture sont nécessaires pour bien comprendre et interpréter tel ou tel document de leur manuel? Il est donc urgent d'intégrer à la formation initiale et continue des enseignants d'histoire, comme de chaque discipline scolaire, des connaissances sur les processus de lecture et sur la fonction épistémique de l'écrit; autrement dit, sur la fonction de l'écrit dans les apprentissages de savoirs.

Certes, les manuels analysés disparaîtront bientôt des classes, remplacés par ceux adoptés dans le cadre de la réforme québécoise des curriculums. Leurs éditeurs et concepteurs ont-ils profité des avancées importantes de la didactique du français, en particulier dans le domaine de la compréhension et de l'interprétation des textes, pour corriger quelques-unes des lacunes constatées? Une lecture superficielle de nouveaux manuels montre qu'ils sont différents. En effet, le texte didactique de base tend à disparaître au profit de très courts textes relativement indépendants, les chapitres sont morcelés en différentes rubriques et l'iconographie gagne du terrain. Cependant, on ne semble toujours pas avoir tenu compte des recherches sur les difficultés de compréhension et d'interprétation des élèves de 13-14 ans. 
On a tendance à confondre textes courts et textes faciles à comprendre, commettant ainsi la grave erreur d'ignorer l'importance de la macrostructure discursive et des liens explicites entre différentes composantes d'un chapitre, et sous-estimant les difficultés propres à une lecture hybride et éclatée. Des recherches (Paret, 1991) montrent en effet que des phrases courtes juxtaposées ne sont pas plus faciles à comprendre que des phrases longues contenant des phrases enchâssées, il en est de même pour une accumulation de petits paragraphes plutôt que la construction de paragraphes plus longs mais présentant une réelle unité sémantique.

Les éditeurs veulent sans doute séduire les élèves en leur proposant les modes de lecture de la toile que ces derniers affectionnent, mais ont-ils mesuré sérieusement la qualité de la compréhension et la capacité de rétention de ce que les élèves lisent sur le web? On peut en douter, car on ne dispose pas encore de suffisamment de données de recherche à ce sujet. Dès lors, dans ce domaine comme dans d'autres, on devrait faire preuve de plus de prudence.

Afin d'évaluer le rôle des manuels dans l'appropriation des connaissances et habiletés disciplinaires, il faudrait compléter la présente étude par une analyse externe portant sur les formes de médiation de l'enseignant entre le manuel et les élèves, et sur l'étayage qu'il leur offre pour assurer leur compréhension et l'interprétation, et une étude quasi expérimentale sur les difficultés précises des élèves à comprendre et à interpréter leur contenus et leurs usages.

Des analyses de ce type seraient nécessaires en raison de l'hétérogénéité des élèves dans les classes, de leur inégalité face à l'écrit (Barré-de Miniac, 1997) et de leurs difficultés importantes en lecture (Fijalkow, 1996; Giasson, 1995b; Soussi, 1995; Sylvanise, 1993). Pourtant, en 1993 déjà, Moniot soulignait que les élèves ne saisissaient que superficiellement le contenu des manuels d'histoire prétendument faciles pour les spécialistes du domaine, et Laparra (1991) montrait que parmi un grand nombre de genres de textes, les textes d'histoire à visée didactique étaient ceux qui posaient le plus de problèmes aux collégiens français de 12-14 ans et qu'ils n'en retenaient que des informations anecdotiques.

ENGLISH TITLE - Competencies needed to understand and interpret Quebec secondary level history textbooks

SUMmARY - This article presents an analysis of the writing in two chapters in two general history textbooks for Quebec students aged 13-14 years old. The objective was to determine the reading competencies required to use these texts as a real learning tool when presented as a teacher substitute. The results show that, without a systematic presentation by teachers for ensuring student comprehension and interpretation, these textbooks cannot serve their role, since there are too many obstacles for young readers.

KEY WORDS • textbooks, history teaching, comprehension, reading, reading competencies.

TíTULO EN ESPAÑOL • Competencias a movilizar para la comprensión y la interpretación de los libros de historia en la secundaria en Quebec 
RESUMEN - Se llevó a cabo un análisis del discurso de dos capítulos de dos libros de texto en historia general, concebidos para alumnos quebequenses de 13-14 años para conocer las competencias que estos alumnos deben de movilizar en lectura para que estos libros de texto constituyan una verdadera herramienta de aprendizaje, con más razón cuando pretenden ser sustitutos del docente. Se destaca que, si los docentes no proporcionan sistemáticamente más información a los alumnos para asegurarse de su comprensión y de su interpretación, estos libros de texto no pueden cumplir con las expectativas dado que presentan demasiados obstáculos para los jóvenes lectores.

PALABRAS CLAVES • libro de texto, enseñanza de la historia, comprensión, lectura, competencia en lectura.

\section{Références}

Aeby, S. (2004). RÉCRÉATURE. Évaluation d'un programme intensif d'enseignement / apprentissage de la lecture destiné à des élèves de $8^{\mathrm{e}}$ année en grande difficulté. Neuchâtel, Suisse: Institut de recherche et de documentation pédagogique (IRDP).

Astolfi, J.-P. (1986). Les langages et l'élaboration de la pensée scientifique. Le français aujourd'hui, 74, 51-57.

Astolfi, J.-P. (1987). Lire dans un manuel: pas si facile pour les élèves! Cahiers pédagogiques, 254-255, 47-50.

Barré-de Miniac, C. (1997). La famille, l'école et l'écriture. Paris, France: Institut national de recherche pédagogique (INRP).

Baudelot, C., Cartier, M. et Detrez, C. (1999). Et pourtant ils lisent... Paris, France: Seuil.

Bernié, J.-P. (2006). Quand les didactiques disciplinaires interpellent la didactique du français: quel traitement du langagier? Quelle cohérence pour la classe de français? Dans É. Falardeau, C. Fisher, C. Simard et N. Sorin (Dir.): Actes du $9^{e}$ colloque de l'Association internationale pour la didactique du français (Cédérom). Lille / Québec, France / Québec: Association internationale de la recherche en didactique du français - AIRDF / Université Laval.

Blaser, C. (2007). Fonction épistémique de l'écrit: pratiques et conceptions d'enseignants de sciences et d'histoire du secondaire. Thèse de doctorat inédite, Université Laval, Sainte-Foy.

Bouchard, C. et Tessier, G. (1994). L'écrit didactique, mode d'emploi. Sainte-Foy, Québec: Télé-université.

Bourbeau, N. (1988). C’est pas lisable! (sic) - La lisibilité des textes didactiques. Bruxelles, Belgique: De Boeck-Wesmael.

Bronckart, J.-P. et Revaz, F. (1988). Mesurer la lisibilité. Une approche typologique. Revue française de pédagogie, 85, 37-46.

Castellani, G.-A. (1995). Bien lire dans toutes les disciplines au collège. Paris, France: Albin Michel.

Chabanne, J.-C. et Bucheton, D. (2002). Parler et écrire pour penser, apprendre et se construire. Paris, France: Presses universitaires de France.

Chartrand, S.-G. et Blaser, C. (2006). Fonction épistémique des genres disciplinaires scolaires: prolégomènes à un champ de recherches. Dans B. Schneuwly et T. ThévenazChristen (Dir.): Le travail de l'enseignant et l'objet enseigné. Le cas du français langue première. Bruxelles, Belgique: De Boeck. 
Chartrand, S.-G., Blaser, C. et Gagnon, M. (2006). Fonction épistémique de l'écrit et genres disciplinaires. Enquête dans les classes d'histoire et de sciences au secondaire québécois. Revue suisse de l'éducation, 2(3), 275-293.

Darras, F. et Delcambre, I. (1986). Savoir, textes et discours: comment ça se joue dans les manuels? (biologie, géologie, géographie). Le français aujourd'hui, 74, 69-88.

Dauphinais, G. (1994). De la préhistoire au siècle actuel (2 édition). Saint-Laurent, Québec: ERPI.

Delcambre, I. (1988). La relation texte/image. Dans Y. Ginsburger-Vogel (Dir.) : Des manuels pour apprendre. Paris, France: Institut national de recherche pédagogique (INRP).

Derry, M. et Vidal, S. (1994). La lisibilité des manuels. Traces, 32(3), 12-18.

Dumortier, J.-L. (1999). Pour composer des questionnaires de compréhension qui favorisent l'autonomie du lecteur. Vie pédagogique, 118, 51-54.

Escalle, J. et Magnan, A. (2003). L'apprentissage de la lecture. Fonctionnement et développement cognitifs. Paris, France: Armand Colin.

Falardeau, É. (2003). Compréhension et interprétation: deux composantes de la lecture littéraire. Revue des sciences de l'éducation, 29(3), 673-694.

Fijalkow, J. (1996). Mauvais lecteurs. Pourquoi? (3édition). Paris, France: Presses universitaires de France.

Fillon, P. et Vérin, A. (2001) Écrire pour comprendre les sciences. Numéro thématique. Aster, 33.

Garon, R. (2004). 20 ans de lecture de livres au Québec. Dans B. Dallaire (Dir.): États des lieux du livre et des bibliothèques. Observatoire de la culture et des communications $d u$ Québec. Québec, Québec: Institut de la statistique du Québec.

Gérard, F.-M. et Roegiers, X. (1993). Concevoir et évaluer des manuels scolaires. Bruxelles, Belgique: De Boeck-Wesmael.

Giasson, J. (1995a). La lecture: de la théorie à la pratique. Boucherville, Québec: Gaëtan Morin Éditeur.

Giasson, J. (1995b). Stratégies de lecture. Programme d'intervention auprès des élèves à risque: une nouvelle option éducative. Boucherville, Québec: Gaëtan Morin Éditeur.

Giasson, J. (2000). La compréhension en lecture. Boucherville, Québec: Gaëtan Morin Éditeur.

Ginsburger-Vogel, Y. (1987). Apprentissages scientifiques au collège et pratiques documentaires. Paris, France: Institut national de recherche pédagogique (INRP).

Ginsburger-Vogel, Y. (1988). Des manuels pour apprendre. Paris, France: Institut national de recherche pédagogique (INRP).

Golder, C. et Goanac'h, D. (1998). Lire et comprendre. Psychologie de la lecture. Paris, France: Hachette.

Goody, J. (1979). La raison graphique. La domestication de la pensée sauvage. Paris, France: Éditions de Minuit.

Grossmann, F. (1999). Littératie, compréhension et interprétation des textes. Numéro thématique. Repères, 19. 
Le Bouffant, M. (1986). Lire pour apprendre: utiliser les textes documentaires. Le français aujourd'hui, 74, 89-99.

Laparra, M. (1991). Problèmes de lecture posés par l'écriture des textes historiques à visée didactique. Pratiques, 69, 97-124.

Lebrun, J. (2006). Le manuel scolaire réformé: quelle place pour la médiation de l'enseignant et les apprentissages des élèves? Dans J. Lebrun, J. Bédard, A. Hasni et V. Grenon (Dir.) : Le matériel didactique et pédagogique: soutien à l'appropriation ou déterminant de l'intervention éducative. Québec, Québec: Presses de l'Université Laval.

Ministère de l'Éducation nationale (1997). La maîtrise de la langue au collège. Paris, France: Centre national de documentation pédagogique - CNDP et Savoir lire.

Ministère de l’Éducation du Québec - MÉQ (1982). Histoire générale, formation générale. Programme d'études, 2 esecondaire. Québec, Québec: Gouvernement du Québec.

Moniot, H. (1993). Didactique de l'histoire. Paris, France: Nathan.

Morais, J. (1994). L'art de lire. Paris, France: Odile Jacob.

Morais, J., Pierre, R. et Kolinsky, R. (2003). Du lecteur compétent au lecteur débutant: implication des recherches en psycholinguistique cognitive et en neuropsychologie pour l'enseignement de la lecture. Revue des sciences de l'éducation, 29(1), 51-100.

Paret, M.-Chr. (1991). La syntaxe écrite des élèves du secondaire. Montréal, Québec: Université de Montréal, Faculté des sciences de l'éducation.

Pierre, R. (2003a, 11 novembre). Pour grimper dans le palmarès des écoles. Avant tout la lecture et l'écriture. Le Devoir.

Pierre, R. (2003b). Introduction. L'enseignement de la lecture au Québec de 1980 à 2000 : fondements historiques, épistémologiques et scientifiques. Revue des sciences de l'éducation, 29(1), 3-36.

Préfontaine, C., Godard, L. et Fortier, G. (1998). Pour mieux comprendre la lecture et l'écriture. Montréal, Québec: Les Éditions Logiques.

René, D. (1991). Apprendre à écrire un devoir d'histoire. Pratiques, 69, 71-95.

Roby, J. et Paradis, C. (1995). Enjeux et découvertes. Tome 1. Laval, Québec: Éditions HRW.

Schneuwly, B. (1995). Apprendre à écrire. Une approche socio-historique. Dans J.-Y. Boyer, J.-P. Dionne et P. Raymond (Dir.): La production de textes. Montréal, Québec: Les Éditions Logiques.

Soussi, A. (1995).Comment lisent-ils en sixième? Une observation des compétences et des stratégies en compréhension de l'écrit chez des élèves romands de sixième. Neuchâtel, Suisse: Institut de recherche et de documentation pédagogique (IRDP).

Sylvanise, S. (1993). Le rapport à la lecture chez des mauvais élèves de sixième. Dans G. Chaveau, M. Remond et E. Rogavgas-Chaveau (Dir.) : L'enfant apprenti lecteur. Paris, France: L'Harmattan.

Vargas, C. (2006). Les manuels scolaires. Imperfections nécessaires, imperfections inhérentes et imperfections contingentes. Dans M. Lebrun (Dir.): Le manuel scolaire. Un outil à multiples facettes. Montréal, Québec: Presses de l'Université du Québec.

Vygotski, L. (1997). Pensée et langage. Paris, France: La Dispute. 
58 Revue des sciences de l'éducation, volume 35, n² 2, 2009

Madame Suzanne Chartrand est membre du Centre de recherche interuniversitaire sur la formation et la profession enseignante (CRIFPE).

\section{Correspondance}

Suzanne.Chartrand@fse.ulaval.ca

Ce texte a été révisé par Christelle Lison.

Texte reçu le: 8 mars 2007

Version finale reçue le: 7 janvier 2008

Accepté le: 22 janvier 2008 